\title{
Mean Tear-Film Lipid Layer Thickness and Video Display Terminal Time as Risk Factors for Abnormal Blinking in Children
}

\author{
Hui Zhao ${ }^{1 *+}$, Shi-Nan Wu ${ }^{2,3+}$, Zhe Cheng ${ }^{1}$, Dong Xiao ${ }^{1}$, Hui-Ye Shu ${ }^{2,3}$, Qian-Min Ge ${ }^{2,3}$, \\ Tian Tian ${ }^{1}$ and Yi Shao ${ }^{2,3 *}$ \\ ${ }^{1}$ Xinhua Hospital Affiliated to Shanghai Jiao Tong University School of Medicine, Shanghai, China, ${ }^{2}$ Department of \\ Ophthalmology, The First Affiliated Hospital of Nanchang University, Jiangxi Province Ocular Disease Clinical Research \\ Center, Nanchang, China, ${ }^{3}$ Department of Radiology, The First Affiliated Hospital of Nanchang University, Jiangxi Province \\ Medical Imaging Research Institute, Nanchang, China
}

OPEN ACCESS

Edited by:

Lei Gu,

Max Planck Institute for Heart and Lung Research, Germany

Reviewed by:

Wensi Tao,

University of Miami Health System,

United States

Fei Dong,

University of Cincinnati, United States

*Correspondence:

Hui Zhao

20887787@qq.com

Yi Shao

freebee99@163.com

tThese authors have contributed equally to this work and share first authorship

\footnotetext{
Specialty section

This article was submitted to

Ophthalmology,

a section of the journal

Frontiers in Medicine
}

Received: 29 September 2021 Accepted: 15 November 2021 Published: 06 December 2021

Citation:

Zhao H, Wu S-N, Cheng Z, Xiao D, Shu H-Y, Ge Q-M, Tian T and Shao Y (2021) Mean Tear-Film Lipid Layer Thickness and Video Display Terminal Time as Risk Factors for Abnormal Blinking in Children Front. Med. 8:785901. doi: 10.3389/fmed.2021.785901
Objective: To explore the risk factors for abnormal blinking in children and the role of the tear-film lipid layer thickness (LLT) as a function of duration of video display terminal (VDT) use in children.

Methods: Children attending the Optometry Clinic of Xinhua Hospital affiliated with Shanghai Jiao Tong University were recruited for the study between June 2019 and June 2020. Time spent viewing a VDT (VDTt) over the previous 6 months was recorded. Incomplete blinking (IB) and blinking rate were measured over a 10 s period using the Lipiview® interferometer (Tear Science, Morrisville, NC, USA), and participants were allocated into groups with normal blinking (NBG, blink rate $<20$ blinks/min) and abnormal blinking (ABG, blink rate $\geq 20$ blinks/min). T-test, chi-square test and Mann-Whitney $U$-test were used to compare the differences in tear film (TF) stability indexes and meibomian gland function indexes between the two groups. Binary logistic analysis was used to analyze the risk factors for abnormal blinking and protective factors related to children's use of VDT, and receiver operating characteristic (ROC) curve analysis was also conducted.

Results: A total of 167 children were included, with no statistically significant differences in age or sex between the two groups. According to the $t$-test, VDTt was significantly higher in ABG than NBG, while TF stability indices including tear break up time, LLT and the height of the tear meniscus, were significantly higher in NBG than ABG $(P<0.001)$. The results also showed better meibomian gland function in NBG than ABG $(P<0.05)$. Binary logistic analysis showed that VDTt is an important risk factor for abnormal blinking, and the average of LLT (AVG) was found to be an important protective factor for children using a VDT for long periods, with a cut-off value of $1.5 \mathrm{~h}$ and $57.5 \mathrm{~nm}$, respectively. ROC curve analysis showed that the area under the curve value of VDTt and AVG was 0.833 and 0.969, respectively $(P<0.001)$.

Conclusion: In children, VDTt is an important risk factor for abnormal blinking, and the AVG is an important protective factor for children using VDT for long periods.

Keywords: children, abnormal blinking, video display terminal time, dry eye, tear film alteration 


\section{INTRODUCTION}

A blink is a coordinated movement of eyelids closing and opening in a natural state. The normal blink rate is $10-15$ blinks/min, each of $0.3-0.4 \mathrm{~s}$ duration, with inter-blink interval of 3-4s. When the blink rate or amplitude exceeds an upper limit, it is considered abnormal. Abnormal blink as a chief complaint in children presenting at the ophthalmology clinic is most commonly secondary to other ocular abnormalities. In addition, some scholars have pointed out that abnormal blinks are closely related to the development of meibomian gland dysfunction (MGD) (1). Therefore, clinical optimization of blinking can effectively improve the symptoms of MGD patients. To date, some studies have shown that video display terminal (VDT) use is an important factor in dry eye (2). China has one of the highest portable VDT utilization rates in the world, with 847 million smart phone users in 2019, an average usage time of $27.9 \mathrm{~h}$ per week, $4 \%$ of users being children under the age of 10 and $16.9 \%$ being $10-19$ years old, indicating that about 177 million children making extensive use of smart phones and VDTs (3). Long periods of VDT use are associated with discomfort symptoms such as dry eyes, ocular foreign body sensation, burning sensation and visual fatigue $(4,5)$. The various eye diseases related to VDT use are summarized in Table 1 (612). Relevant studies have shown that dry eye patients often have ocular surface epithelial damage, abnormal blinking and other clinical symptoms. Therefore, children may be at risk of abnormal blinking and possibly MGD (13) after prolonged use of VDT. Fenga et al. reported that long-term VDT users accompanied by MGD were more likely to have symptoms of eye discomfort and go to ophthalmology (11), which was consistent with the results of other scholars' studies (14).

There are many other external factors that can cause abnormal blinking. It has been reported that the main factors determining blink frequency are stimuli external to the eye, such as the state of the tear film (TF) on the surface of the cornea and conjunctiva, the excitation state of relevant receptors and environmental factors (15). As the outermost layer between the eye and the external environment, the ocular lipid layer of the TF plays an important role in reducing tear evaporation, increasing the stability of the TF and promoting the formation of TF on the ocular surface. A series of studies have shown a significant correlation between dry eye symptoms and tear-film lipid layer thickness (LLT) (16). Previous studies have focused on dry eyes following VDT use in adults, but few reports on meibomian glands (MGs) have involved long-term use of VDT in children. In addition, the scale of children's use of VDTs in China is expanding, and the age at which children begin to use VDTs is increasingly early. Children are unable to accurately describe their subjective symptoms, so conditions such as MGD may be neglected in children with long-term use of VDT. Abnormal blinking is a sign which can be observed by the clinician and does not need to be reported by the child. However, there are no published reports of the correlation between abnormal blinking and the time spent using VDT (VDTt), and there is a lack of research on the relationship between LLT, dry eye and abnormal blinking. Through the analysis of the correlation among VDT, dry eye and abnormal blinking, to provide guidance for the prevention and control of abnormal blink and dry eye in advance. Therefore, the purpose of this study was to explore the correlation between blink abnormalities in children and VDTt, the functional state of the MGs and the TF stability represented by LLT.

\section{METHODS}

\section{Patients and Examination}

A total of 167 children aged 15 years or less attending ophthalmology outpatient clinics at Xinhua Hospital Affiliated to Medical College of Shanghai Jiao Tong University between June 2019 and June 2020 were enrolled. The blink rate was measured using the Lipiview $\AA$ interferometer, and subjects with a blink rate of $\geq 20$ blinks/min were allocated to an abnormal blinks group $(\overline{A B G})$, while those with a blink rate of $<20$ blinks/min were allocated to a normal blinks group (NBG) (17). Some data results were shown as mean \pm standard deviation.

Participants were eligible if they met the following criteria: (1) no active inflammation of the eye and no eye drops used within the preceding three months; (2) no history of wearing corneal contact lenses, no history of laser or other ocular surgical operations, no history of ocular trauma, ocular chemical injury or burns; (3) no upper respiratory tract infection within the preceding two weeks; (4) no use of drugs affecting tears such as atropine, neostigmine or artificial tears within the last six months.

Children older than 15 years or with any of the following were excluded: (1) other ocular diseases that may cause ocular surface abnormalities such as abnormal eyelid position, proptosis, or

TABLE 1 | A variety of eye diseases caused by VDT.

\begin{tabular}{|c|c|c|c|}
\hline References & Year & Corresponding population & Eye diseases \\
\hline Moon et al. (6) & 2016 & Children & Dry eye \\
\hline Ranasinghe et al. (7) & 2016 & Computer office workers & Computer Vision Syndrome \\
\hline Basso et al. (8) & 2006 & VDT workers & Myopia \\
\hline Ozawa et al. (9) & 2015 & Office workers aged $20-40$ years & Eye fatigue \\
\hline Lee et al. (10) & 2016 & Myopic patients with open-angle glaucoma & Visual field progression of open-angle glaucoma \\
\hline Fenga et al. (11) & 2008 & VDT workers & Meibomian gland dysfunction \\
\hline Chen et al. (12) & 2017 & Long term VDT user & Paracentral acute middle maculopathy \\
\hline
\end{tabular}

VDT, video display terminal. 
TABLE 2 | General information.

\begin{tabular}{|c|c|c|c|}
\hline Parameters & NBG & ABG & $P$ value ${ }^{c}$ \\
\hline Age (years) ${ }^{a}$ & $7.82 \pm 2.66$ & $8.14 \pm 3.01$ & 0.462 \\
\hline Gender $(F: M)^{b}$ & & & 0.941 \\
\hline Female & 40 (48\%) & 40 (48\%) & \\
\hline Male & 43 (52\%) & $44(52 \%)$ & \\
\hline Blinking rate ${ }^{a}$ & $14.60 \pm 4.00$ & $30.00 \pm 7.62$ & $<0.001$ \\
\hline Incomplete blinking rate ${ }^{a}$ & $13.95 \pm 7.20$ & $23.71 \pm 11.44$ & $<0.001$ \\
\hline VDTt (hours) ${ }^{a}$ & $1.03 \pm 0.95$ & $2.48 \pm 1.08$ & $<0.001$ \\
\hline
\end{tabular}

$P<0.05$ indicates statistical significance.

aIndependent sample $T$ test.

${ }^{b}$ Chi-squared test.

${ }^{c}$ Comparison between the NBG and ABG.

Data showed as mean \pm standard deviation or $n$.

NBG, normal blinking group; ABG, abnormal blinking group; VDTt, video display terminal time.

pterygium; (2) other systemic diseases such as hyperthyroidism that affect tear production; or (3) developmental abnormalities. The study was compliant with the tenets of the Declaration of Helsinki, was approved by the Xinhua Hospital ethics committee and was registered with the Chinese Clinical Trial Registry (trial registration number: ChiCTR2000038908 and the approved number: XHEC-D-2018-103). Prior to each subject's participation, a guardian signed a declaration of paper informed consent.

Eye examination and other procedures were conducted in the following order: (1) The child and/or guardian was asked about the child's duration of VDT use over the preceding six months; (2) Lipiview $\AA$ measurements (including LLT and blink rate) were recorded; (3) tear meniscus height (TH) was measured and MG morphology was assessed using the Oculus Keratography 5M; (4) an ocular surface slit lamp examination was carried out to assess conjunctival congestion and fluorescein BUT, the Marx line (ML) score, MG expression and Meibum score, and corneal fluorescein staining score. All the examinations were finished in one day by the same ophthalmologist in the same dark room.

\section{Measurement of Tear-Film Lipid Layer Thickness (LLT), Incomplete Blinks (IB) and Blink Rate}

The LLT of the TF (minimum, maximum and average), number of incomplete blinks (IB) and the blink rate over a $10 \mathrm{~s}$ period were measured using a Lipiview $\AA$ interferometer and during this procedure the child was encouraged to blink naturally (18). The equipment can automatically calculate the number of blinks per min and the number of abnormal blinks per min.

\section{TH and MG Morphology by Oculus Keratography $5 \mathrm{M}$}

The $\mathrm{TH}$ was measured using the Oculus Keratograph $5 \mathrm{M}$ camera and related software (19). TH $\geq 0.2 \mathrm{~mm}$ indicates normal tear secretion, while $\mathrm{TH}<0.2 \mathrm{~mm}$ indicates insufficient tear secretion. The upper and lower eyelids were inverted, the conjunctiva was fully exposed, and the images were recorded, analyzed and height calculated. MG dropout was scored using the same equipment according to the following scale: I (normal, with no MG deficiency); II (MG deficiency < 1/3); III (MG deficiency 1/3-2/3); IV (MG deficiency > 2/3) (20).

\section{Slit Lamp Examination}

To measure TF breakup time (BUT), $2 \mu \mathrm{L} 1 \%$ sodium fluorescein preservative free solution was dropped into the lower conjunctival sac and the participant was instructed to blink several times for a few seconds. TBUT was measured three times, and the mean calculated.

Corneal fluorescence staining score (FL) was measured by considering the corneal surface as four quadrants: supra-nasal, infra-nasal, superior temporal, and inferior temporal, with each quadrant being scored I-IV for a total out of 12 points. Scoring criteria were: I (no staining; 0 point); II (mild scattered spot staining, $1-30$ spots stained; 1 point); III (moderate staining, $>30$ spots stained but staining not fused; 2 points); IV (heavy staining or sheet staining of the entire cornea, spot staining fused, with filaments, or ulcers; 3 points) (21).

The Marx line (ML) score was calculated for the outer, middle, and inner thirds of the lower eyelid margin, and recorded as: I [entirely on the conjunctival side of the meibomian orifices (MOs)]; II (part of the ML touches the MOs); III (ML runs through all of the MOs); and IV (ML runs on the eyelid margin side of the MOs) (22-24).

To measure MG expression (MGE), the five glands at the center of the upper and lower eyelids were located and their orifices observed. Scores were based on the following scale: I (normal, meibum secretion from all glands with light pressure on the eyelid); II (secretion from 3 to 4 glands with light pressure on the eyelid; III (secretion from 1 to 2 glands with light pressure on the eyelid); IV: no secretion from any glands with light pressure on the eyelid) (25).

The quality of secreted meibum was scored (Meibum score) according to the following criteria: I (normal, clear, transparent lid ester); II (cloudy lid ester); III (cloudy lid ester with granules); IV (thick lid fat with a toothpaste-like consistency) (26).

\section{Statistical Analyses}

Statistical analysis was conducted using SPSS (version 25.0) and $\mathrm{R}$ (version 4.0.5; package pROC version 1.18.0; package ggplot2 version 3.3.5) to carry out $t$-tests, Chi-square test and MannWhitney $U$ test between groups. Binary logistic analysis was performed on the related factors according to the grouping. Receiver operating characteristic (ROC) curves and the area under the curve (AUC) were used to analyze sensitivity and specificity of risk factors as diagnostic indicators. $P$ values of $<0.05$ were considered statistically significant.

\section{RESULTS}

\section{Patient Demographics}

A total of 167 children aged 15 years or younger were included in this case-control study. And 167 right eyes were examined in the study. The NBG included 40 females and 43 males (mean age $7.82 \pm 2.66$ years; range $2-15$ years) and the ABG included 40 


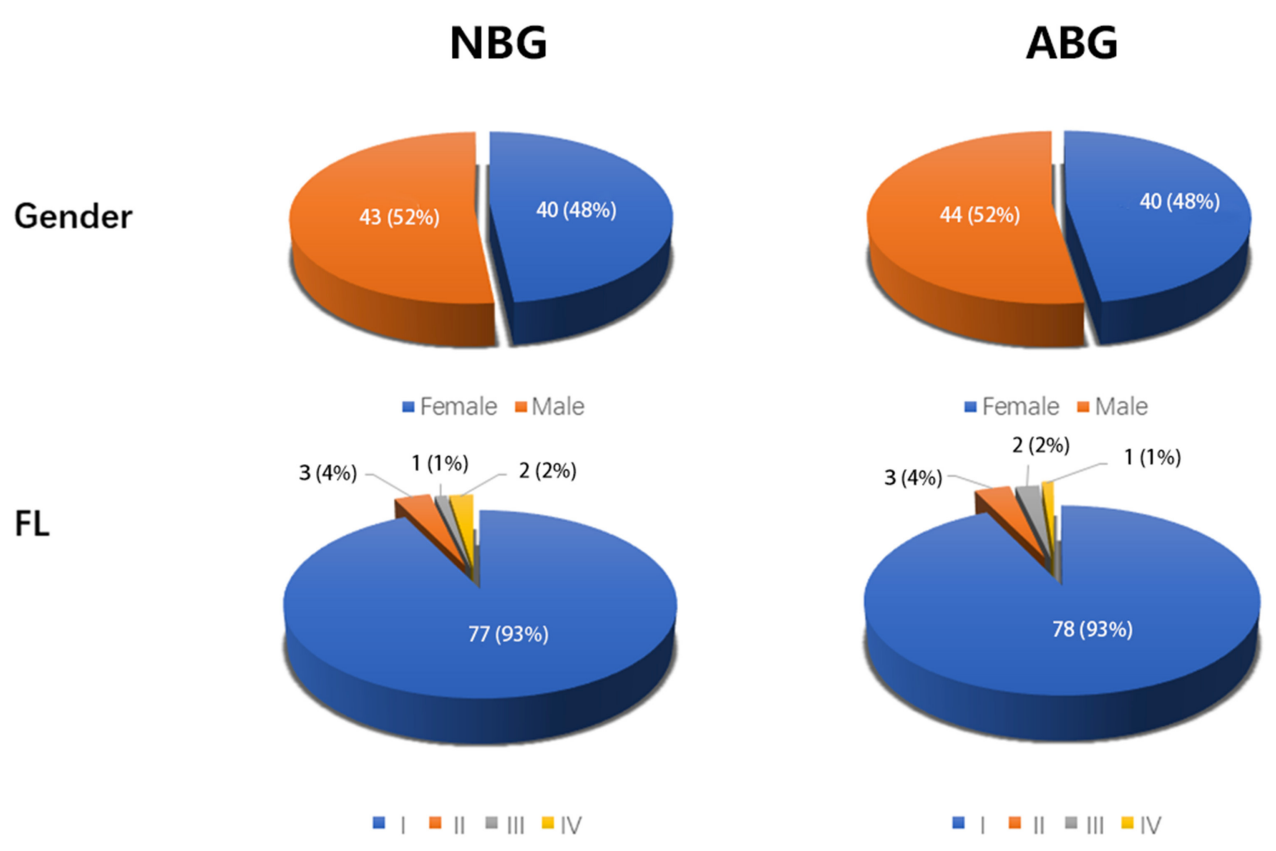

FIGURE 1 | Clinical characteristics of NBG and ABG. N = 83 in NBG, $n=84$ in ABG. NBG, normal blinking group; ABG, abnormal blinking group; FL, corneal fluorescence staining score.

TABLE 3 | The index of tear film.

\begin{tabular}{lcccc}
\hline Parameters & NBG & ABG & $\mathbf{Z}^{\mathbf{c}}$ & $\boldsymbol{P}_{\text {value }}$ \\
\hline $\mathrm{FL}^{\mathrm{a}}$ & & -0.032 & 0.974 & \\
I & $77(93 \%)$ & $78(93 \%)$ & & \\
II & $3(4 \%)$ & $3(4 \%)$ & & \\
III & $1(1 \%)$ & $2(2 \%)$ & & \\
IV & $2(2 \%)$ & $1(1 \%)$ & & \\
BUT(s) & $6.25 \pm 2.63$ & $4.79 \pm 2.65$ & - & $<0.001$ \\
TH $(\mathrm{mm})^{\mathrm{b}}$ & $0.21 \pm 0.08$ & $0.18 \pm 0.07$ & - & $<0.001$ \\
AVG & $70.70 \pm 22.05$ & $47.70 \pm 16.88$ & - & $<0.001$ \\
MIN $^{\mathrm{b}}$ & $63.81 \pm 22.33$ & $42.58 \pm 16.40$ & - & $<0.001$ \\
MAX $^{\mathrm{b}}$ & $81.25 \pm 21.27$ & $59.35 \pm 20.18$ & - & $<0.001$ \\
\hline
\end{tabular}

$P<0.05$ indicates statistical significance.

a Mann-Whitney $U$ test.

${ }^{b}$ Independent sample $T$ test.

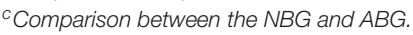

Data showed as mean \pm standard deviation or $n$.

NBG, normal blinking group; ABG, abnormal blinking group; FL, corneal fluorescence staining score; BUT, tear film breakup time; TH, tear meniscus height; AVG, the average of tear film lipid layer thickness; MIN, the minimum of tear film lipid layer thickness; MAX, the maximum of tear film lipid layer thickness.

females and 44 males (mean age $8.14 \pm 3.01$ years; range $2-15$ years). The two groups were statistically similar in age and gender ( $P=0.462$ and $P=0.941$ respectively). The mean blink rate in the NBG was $14.60 \pm 4.00$, and this was significantly lower than the ABG blink rate of $30.00 \pm 7.62(P<0.001)$. In addition, the frequency of incomplete blinking in $\mathrm{ABG}(23.71 \pm 11.44)$ was significantly higher than that in NBG $(13.95 \pm 7.20 ; P<0.001)$.
The VDTt was also significantly different between the two groups, with longer times in ABG than in NBG $(P<0.001)$. Data are shown in Table 2; Figure 1.

\section{Tear Film Stability}

The TH measure provided an indication of the quantity of TF. The TH and BUT of ABG was significantly lower than NBG, (TH $0.18 \pm 0.07$ and $0.21 \pm 0.08$; BUT $4.79 \pm 2.65$ and 6.25 $\pm 2.63 ; P<0.001$ ) (Table 3). LLT was assessed using mean and range. All LLT indices were significantly lower in ABG than NBG (mean $47.70 \pm 16.88$ and $70.70 \pm 22.05$; minimum $42.58 \pm 16.40$ and $63.81 \pm 22.33$; and maximum $59.35 \pm 20.18$ and $81.25 \pm$ 21.27, respectively; all $P<0.001)$. No significant between-groups difference was found in FL $(Z=-0.032, P=0.974$, Table 3) (Figure 2).

\section{Meibomian Gland Function}

MG dropout, meibum score, MGE and ML were used to evaluate the morphology and function of MG. Although there was no significant difference in MG dropout between the two groups ( $Z$ $=-0.012, P=0.990)$. $\mathrm{ML}(Z=-3.886, P<0.001)$, MGE $(Z$ $=-3.798, P<0.001)$ and Meibum scores $(Z=-2.411, P=$ $0.016)$ were lower in the NBG than in the ABG. More detailed information is shown in Table 4; Figure 3.

\section{Binary Logistic and ROC Curve Analysis}

Binary logistic multivariate analysis based on ABG and NBG grouping showed that VDTt was an important risk factor for abnormal blinking. Based on an odds ratio of 3.486, it can be inferred that the risk of abnormal blinking after long-term use 


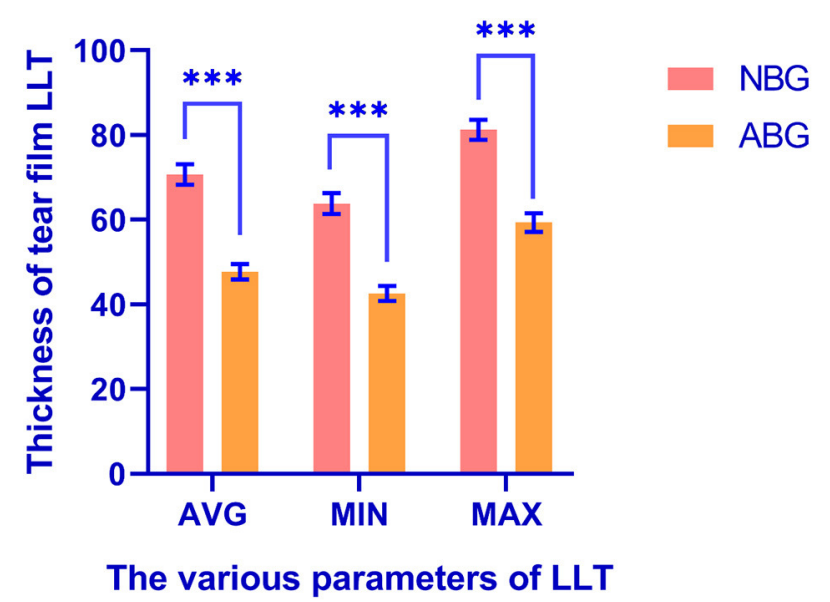

FIGURE 2 | The difference of tear film LLT between NBG and ABG. ${ }^{* \star *}$ There was a significant difference between the two groups. LLT, lipid layer thickness; NBG, normal blinking group; ABG, abnormal blinking group; AVG, the average of tear film lipid layer thickness; MIN, the minimum of tear film lipid layer thickness; MAX, the maximum of tear film lipid layer thickness.

TABLE 4 | The function of meibomian gland.

\begin{tabular}{|c|c|c|c|c|}
\hline Parameters & NBG & ABG & $\mathbf{Z}^{\mathbf{b}}$ & $P$ value $^{b}$ \\
\hline $\mathrm{ML}^{\mathrm{a}}$ & & & -3.886 & $<0.001$ \\
\hline I & 59 (71\%) & 36 (43\%) & & \\
\hline$\|$ & 3 (4\%) & $5(6 \%)$ & & \\
\hline III & 21 (25\%) & 36 (43\%) & & \\
\hline IV & 0 (0\%) & 7 (8\%) & & \\
\hline $\mathrm{MGE}^{\mathrm{a}}$ & & & -3.798 & $<0.001$ \\
\hline I & $51(61 \%)$ & 28 (33\%) & & \\
\hline ॥ & 22 (27\%) & 34 (40\%) & & \\
\hline III & 10 (12\%) & $18(21 \%)$ & & \\
\hline IV & $0(0 \%)$ & $4(5 \%)$ & & \\
\hline MG dropout ${ }^{\mathrm{a}}$ & & & -0.012 & 0.990 \\
\hline I & 81 (98\%) & 82 (98\%) & & \\
\hline ॥ & $0(0 \%)$ & $1(1 \%)$ & & \\
\hline III & $2(2 \%)$ & $0(0 \%)$ & & \\
\hline IV & $0(0 \%)$ & 1 (1\%) & & \\
\hline Mebium score ${ }^{a}$ & & & -2.411 & 0.016 \\
\hline I & 80 (97\%) & 72 (86\%) & & \\
\hline ॥ & $2(2 \%)$ & 7 (8\%) & & \\
\hline III & 1 (1\%) & $5(6 \%)$ & & \\
\hline
\end{tabular}

$P<0.05$ indicates statistical significance. a Mann-Whitney U test.

${ }^{b}$ Comparison between the NBG and $A B G$.

NBG, normal blinking group; $A B G$, abnormal blinking group; $M L$, marx line; MGE, meibomian gland expression; MG dropout, meibomian gland dropout degree.

of VDT is 3.486 times higher than that of people who have not used VDT for a long time. ROC curve analysis was used to further explore this risk factor, and showed an AUC value for VDTt of 0.833. Based on the ROC curve, cut-off value of 1.5 , sensitivity

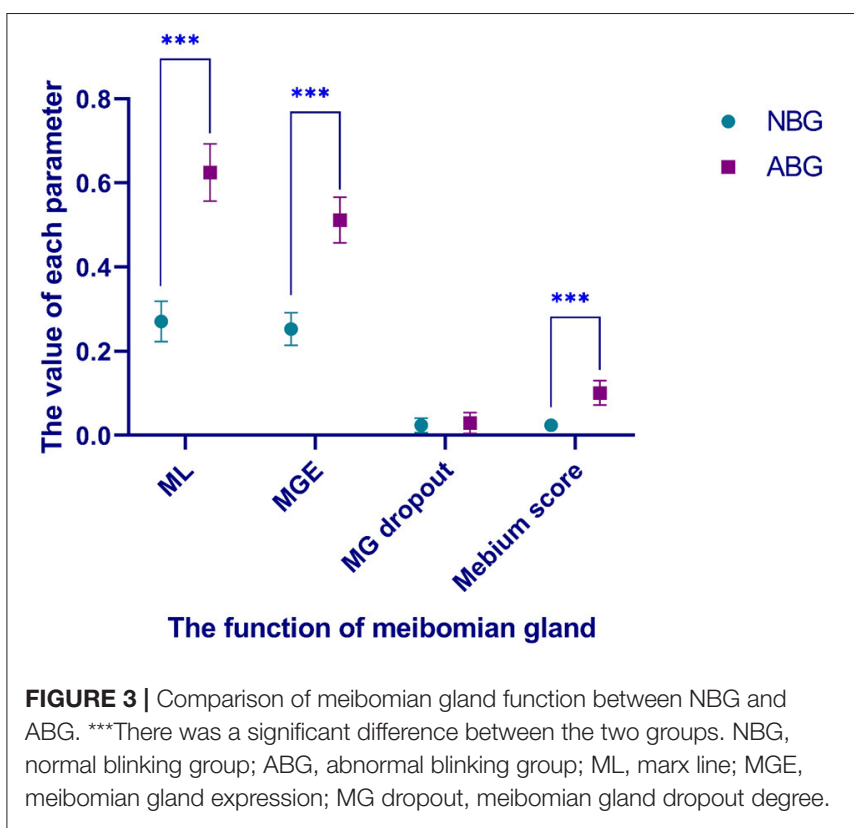

of 0.827 , and specificity of 0.825 were determined $(P<0.001)$ (Tables 5, 6; Figure 4A).

\section{Binary Logistic Analysis Results and ROC Curve With $1.5 \mathrm{~h}$ as Boundary-Value Grouping}

In order to exclude the collinearity effect of VDTt on other covariates, data were grouped based on the $1.5 \mathrm{~h}$ cut off value, and binary logistic multivariate analysis was conducted. Mean LLT was found to be an important protective factor with an odds ratio of 0.762 . To evaluate the clinical significance of mean LLT in differential diagnosis, further ROC curve analysis was performed. The AUC value corresponding to mean LLT was 0.969 , with cutoff value of 57.5 , sensitivity and specificity of 0.857 and 0.940 respectively $(P<0.001)$ (Tables 5, 6; Figure 4B).

\section{DISCUSSION}

The present study identified the amount of time spent using a VDT as an important risk factor for abnormal blinking in children, and that VDTt of $<1.5 \mathrm{~h}$ per day may be more conducive to children's eye health than longer periods. Moreover, statistical analysis pointed to mean LLT as an important protective factor for long periods of VDT use, with a bound value of $57.5 \mathrm{~nm}$. This indicates that mean LLT $\leq 57.5 \mathrm{~nm}$ found in clinical ophthalmological examination would require consideration of the child's use of VDT and its possible impact on TF stability (Figure 5). According to the above clinical indicators, ophthalmologists could play a further important role in guiding children's eye health.

Previous research has shown that prolonged use of VDT could cause musculoskeletal pain, poor mental performance and psychological distress, and most commonly eye irritation (27). 
TABLE 5 | Binary logistic analysis results under different groups.

\begin{tabular}{|c|c|c|c|c|c|}
\hline Factor & Cut-Off value & Sensitivity (\%) & Specificity (\%) & AUC & $P$ value \\
\hline VDTta & 1.5 & 82.7 & 82.5 & 0.833 & $<0.001$ \\
\hline $\mathrm{AVG}^{\mathrm{b}}$ & 57.5 & 85.7 & 94.0 & 0.969 & $<0.001$ \\
\hline
\end{tabular}

a The results are grouped according to a bound of 20 blinks per min.

${ }^{b}$ The results are grouped according to a daily bound value of $1.5 \mathrm{~h}$ of VDT usage.

$P<0.05$ denoted statistical significance.

B, coefficient of regression; OR, odds ratio; Cl, confidence interval; VDTt, video display terminal time; AVG, the average of tear film lipid layer thickness.

TABLE 6 | The Cut-off Value, sensitivity, specificity, and AUC of factors for the different grouping conditions.

\begin{tabular}{|c|c|c|c|c|}
\hline Factors & B & OR & OR $(95 \% \mathrm{Cl})$ & $P$ value \\
\hline VDTt ${ }^{a}$ & 1.249 & 3.486 & $2.410-5.042$ & $<0.001$ \\
\hline$A V G^{b}$ & -0.272 & 0.762 & $0.687-0.845$ & $<0.001$ \\
\hline
\end{tabular}

a The results are grouped according to a bound of 20 blinks per min.

${ }^{b}$ The results are grouped according to a daily bound value of $1.5 \mathrm{~h}$ of VDT usage.

$P<0.05$ denoted statistical significance.

VDTt, video display terminal time; AVG, the average of tear film lipid layer thickness; AUC, area under the curve.
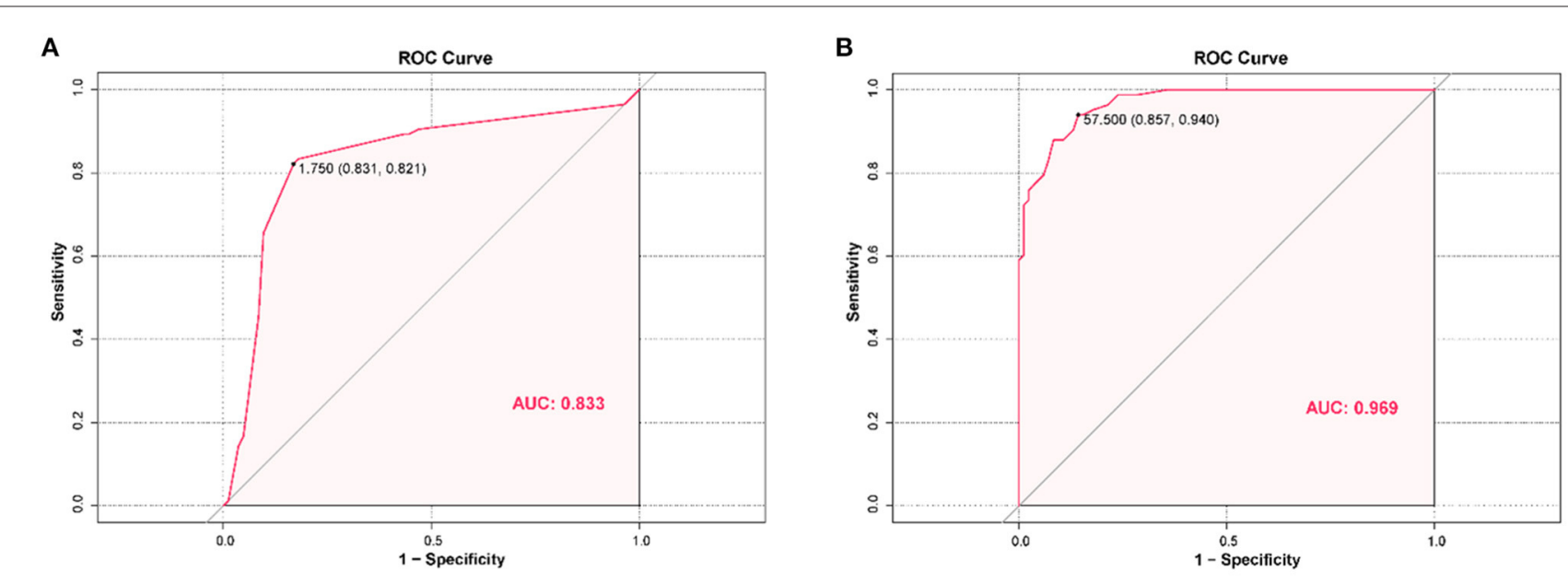

FIGURE 4 | (A) The receiver operating characteristics (ROC) curves of risk factors for detecting children with abnormal blinking. ROC curves of VDTt showed that the AUC value was $0.833(P<0.001)$. The sensitivity and specificity were 82.7 and $82.5 \%$, respectively. (B) The receiver operating characteristics (ROC) curves for factors associated with prolonged use of VDT in children. ROC curves of AVG showed that the AUC value was $0.969(P<0.001)$. The sensitivity and specificity were 85.7 and $94.0 \%$, respectively. VDTt, video display terminal time; AVG, the average of tear film lipid layer thickness; AUC, area under the curve.

In China, a high risk factor for myopia in children is the use of VDT in close proximity, especially long periods of smartphone use (28). However, to date, little research exists on the correlation between abnormal blinking and the use of VDT. Since it is difficult for children to clearly express their symptoms, and children with xerophthalmia may have allergic conjunctivitis to varying degrees, these diseases may be misdiagnosed clinically (29). In a prospective study of frequent blinking in children, the most common cause was abnormality of the anterior segment or eyelid (30), but diverse causative factors exist and most are related to benign or self-limiting conditions (see Figure 6) (31-33). Careful medical history and clinical examination are important to determine the corresponding etiology and to provide timely and targeted treatment.

In this study, a significant risk factor for excessive blinking in children was prolonged video device use. Some scholars have pointed out that the prolonged use of VDT is an important cause of dry eyes, and found that warming moist chamber goggles have an excellent curative effect (34). There is a time-dependent oxidative stress response in ocular surface tissue, but the VDT durations in the present study are not sufficient to discern any damage to the ocular surface. Longer periods of VDT use may cause damage to the ocular surface. Perhaps short periods of use cause the decline of MG secretion function and the thinning of 


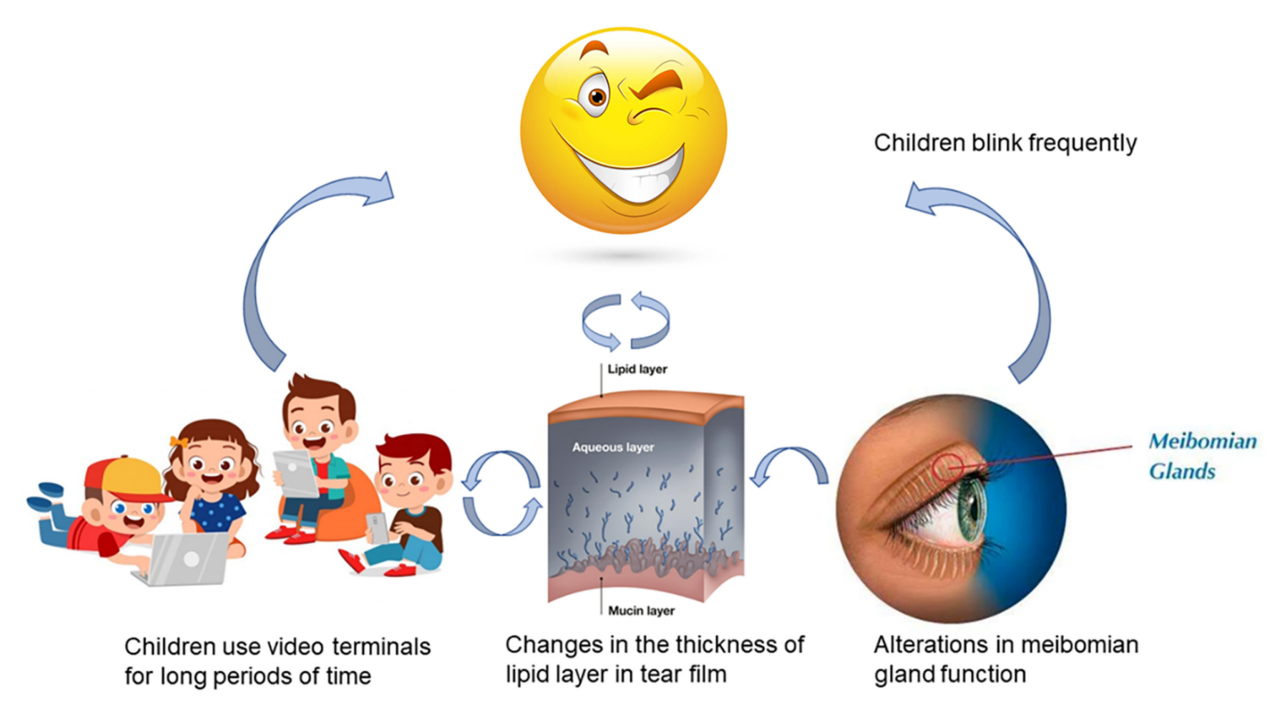

FIGURE 5 | Frequent blinking in children was associated with prolonged use of terminal devices, thickness of tear film lipid layer, and alterations in meibomian gland function.

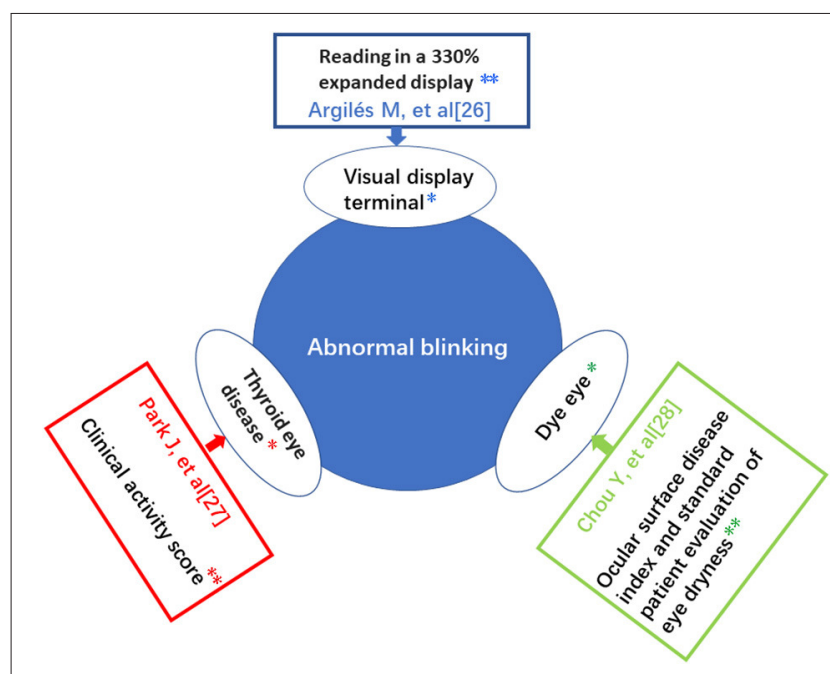

FIGURE 6 | Summary of risk factors leading to abnormal blinking and their important influencing factors. ${ }^{*}$ Risk factors. ${ }^{\star \star}$ Important influencing factors.

TF lipid layer, which leads to the deterioration of TF stability, but not the loss of MGs.

Numerous studies have shown reduced blink rates in longterm VDT users, as low as 7-11 blinks/min, with a significant increase in the percentage of incomplete blinks $(35,36)$. In children, with highly appealing entertainment and materials available to view on VDTs, blink rate is lower still, with the orbicularis oculi and Riolan muscles in children contracting completely around the eyelid gland opening. MG acini constantly synthesize and secrete meibum, which leads to the abnormal discharge of meibum, a decrease in MG secretion capacity and obstruction of the meibum $(37,38)$. This may be alleviated by the patient squeezing the eyes hard and increasing blink frequency. Previous research has shown that $60 \%$ of people who use VDT for long periods have significantly decreased blink rates, which in turn can lead to MGD (39). We hypothesize that children are more sensitive than adults to MG and TF abnormalities, with associated increased blink rate and abnormal blinking habits, and these signs can be misdiagnosed by clinicians as Tourette's syndrome.

Interestingly, studies have shown that incomplete blinking is associated with a two-fold increased risk of dry eye disease in patients with poor LLT (40). The TF lipid layer is composed of lipids secreted by meibomian glands, is located in the outermost layer of the TF, and has thickness in the range of $20-180 \mathrm{~nm}$. As the lipid contact surface between the eye and the external environment, TF lipid layer can assist other TF components to inhibit tear evaporation, stabilize the air-tear surface between eye opening and blinks, and play a role in the first line of defense against bacterial invasion (41). The thickness of TF lipid layer is affected by a decrease of eyelid lipid or a change of tear composition.

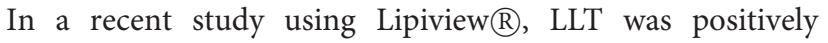
correlated with the number of normally secreting MGs, with LLT $\leq 60 \mathrm{~nm}$ in adults with dry eye, indicating a clinical application of MG assessment (33). This evaluation method has important clinical application value. In our study, BUT, TH and LLT were significantly lower in ABG than NBG. These finding suggest poor TF stability and a possibility of evaporative dry eye in children with abnormal blinking. For those using VDT for long periods, maintaining appropriate LLT could be a significant protective factor for ocular health. According to our statistical analysis, when the average LLT is reduced to $57.5 \mathrm{~nm}$ or less, TF stability may be significantly reduced in children using VDTs.

There are some limitations to our study. Firstly, abnormal blinking was assessed using the blink rate and number of 
incomplete blinks, and excessive blinking was not studied. Secondly, ocular surface morphology was observed over a short period, and longer observation time would help to compare different research results and support the research conclusion. Thirdly, the sample size of each group in the study was small, and larger studies would provide more certainty about the effect of VDTt on TF stability in children. Finally, the children involved in this study were all from the eastern Chinese city of Shanghai, so it is difficult to exclude the effect of air pollution on the ocular surface (42).

\section{DATA AVAILABILITY STATEMENT}

The original contributions presented in the study are included in the article/supplementary materials, further inquiries can be directed to the corresponding author/s.

\section{ETHICS STATEMENT}

The studies involving human participants were reviewed and approved by Xinhua Hospital Affiliated to Medical College of Shanghai Jiao Tong University. Written informed consent to

\section{REFERENCES}

1. Wan $\mathrm{T}$, Jin $\mathrm{X}$, Lin $\mathrm{L}, \mathrm{Xu} \mathrm{Y}$, Zhao $\mathrm{Y}$. Incomplete blinking may attribute to the development of meibomian gland dysfunction. Curr Eye Res. (2016) 41:179-85. doi: 10.3109/02713683.2015.1007211

2. Tsubota K, Yokoi N, Shimazaki J, Watanabe H, Dogru M, Yamada $M$ et al. New perspectives on dry eye definition and diagnosis: a consensus report by the Asia dry eye society. Ocul Surf. (2017) 15:65-76. doi: 10.1016/j.jtos.2016.09.003

3. The 45th China Statistical Report on Internet Development. China Internet Network Information Center.

4. Nakamura S. Approach to dry eye in video display terminal workers (basic science). Invest Ophthalmol Vis Sci. (2018) 59:DES130-7. doi: 10.1167 /iovs.17-23762

5. Mehra D, Galor A. Digital screen use and dry eye: a review. Asia Pac J Ophthalmol (Phila). (2020) 9:491-7. doi: 10.1097/APO.0000000000000328

6. Moon JH, Kim KW, Moon NJ. Smartphone use is a risk factor for pediatric dry eye disease according to region and age: a case control study. $B M C$ Ophthalmol. (2016) 16:188. doi: 10.1186/s12886-016-0364-4

7. Ranasinghe P, Wathurapatha WS, Perera YS, Lamabadusuriya DA, Kulatunga $\mathrm{S}$, Jayawardana $\mathrm{N}$, et al. Computer vision syndrome among computer office workers in a developing country: an evaluation of prevalence and risk factors. BMC Res Notes. (2016) 9:150. doi: 10.1186/s13104-016-1962-1

8. Basso A, Di Lorenzo L, Cramarossa AA, Corfiati M, Ria W, Bellino R, et al. Evaluation of myopia in a group of people working with video terminals: first results. G Ital Med Lav Ergon. (2006) 28:207-9.

9. Ozawa Y, Kawashima M, Inoue S, Inagaki E, Suzuki A, Ooe E, et al. Bilberry extract supplementation for preventing eye fatigue in video display terminal workers. J Nutr Health Aging. (2015) 19:548-54. doi: 10.1007/s12603-014-0573-6

10. Lee JR, Lee J, Lee JE, Lee JY, Kook MS. Optic disc tilt direction affects regional visual field progression rates in myopic eyes with openangle glaucoma. Graefes Arch Clin Exp Ophthalmol. (2016) 254:2267-76. doi: 10.1007/s00417-016-3501-0

11. Fenga C, Aragona P, Cacciola A, Spinella R, Di Nola C, Ferreri F, et al. Meibomian gland dysfunction and ocular discomfort in video display terminal workers. Eye (Lond). (2008) 22:91-5. doi: 10.1038/sj.eye.6703025 participate in this study was provided by the participants' legal guardian/next of kin.

\section{AUTHOR CONTRIBUTIONS}

$\mathrm{HZ}$ and S-NW were involved in the data curation and writing of the original draft. ZC, S-NW, and DX performed the data curation and formal analysis and participated in the writing and editing of the manuscript. H-YS was involved in the study conceptualization and methodology design. Q-MG and TT were involved in data validation and visualization. YS and $\mathrm{HZ}$ were involved in the study conceptualization, data curation, funding acquisition, and project administration. All authors have read and approved the final manuscript.

\section{FUNDING}

This study was supported in part by the Medicine \& Engineering Collaboration Research Fund of Shanghai Jiao Tong University (ZH2018QNB27). The funders have no role in the study design, data collection and analysis, decision on publishing, or preparation of the manuscript.

12. Chen Y, Hu Y. The optical imaging of idiopathic paracentral acute middle maculopathy in a Chinese young man and review of the literature. Photodiagn Photodyn Ther. (2017) 19:383-7. doi: 10.1016/j.pdpdt.2017.07.004

13. Talens-Estarelles C, García-Marqués JV, Cervino A, García-Lázaro S. Use of digital displays and ocular surface alterations: a review. Ocul Surf. (2021) 19:252-65. doi: 10.1016/j.jtos.2020.10.001

14. Wu H, Wang Y, Dong N, Yang F, Lin Z, Shang X, et al. Meibomian gland dysfunction determines the severity of the dry eye conditions in visual display terminal workers. PLoS ONE. (2014) 9:e105575. doi: 10.1371/journal.pone.0105575

15. Collins MJ, Iskander DR, Saunders A, Hook S, Anthony E, Gillon R. Blinking patterns and corneal staining. Eye Contact Lens. (2006) 32:287-93. doi: 10.1097/01.icl.0000224551.58399.9a

16. Foulks GN. The correlation between the tear film lipid layer and dry eye disease. Surv Ophthalmol. (2007) 52:369-74. doi: 10.1016/j.survophthal.2007.04.009

17. Doughty MJ, Naase T. Further analysis of the human spontaneous eye blink rate by a cluster analysis-based approach to categorize individuals with 'normal' versus 'frequent' eye blink activity. Eye Contact Lens. (2006) 32:294-9. doi: 10.1097/01.icl.0000224359.32709.4d

18. Gulati S, Jain S. Ocular pharmacology of tear film, dry eye, and allergic conjunctivitis. Handb Exp Pharmacol. (2017) 242:97-118. doi: 10.1007/164_2016_73

19. Pérez Bartolomé F, Martínez de la Casa JM, Arriola Villalobos P, Fernández Pérez C, Polo V, Sánchez Jean R, et al. Ocular redness measured with the keratograph $5 \mathrm{M}$ in patients using anti-glaucoma eye drops. Semin Ophthalmol. (2018) 33:643-50. doi: 10.1080/08820538.2017. 1395891

20. Wong S, Srinivasan S, Murphy PJ, Jones L. Comparison of meibomian gland dropout using two infrared imaging devices. Cont Lens Anterior Eye. (2019) 42:311-7. doi: 10.1016/j.clae.2018.10.014

21. Amparo F, Wang H, Yin J, Marmalidou A, Dana R. Evaluating corneal fluorescein staining using a novel automated method. Invest Ophthalmol Vis Sci. (2017) 58:BIO168-73. doi: 10.1167/iovs.17-21831

22. Schiffman RM, Christianson MD, Jacobsen G, Hirsch JD, Reis BL. Reliability and validity of the ocular surface disease index. Arch Ophthalmol. (2000) 118:615-21. doi: 10.1001/archopht.118.5.615 
23. Yamaguchi M, Kutsuna M, Uno T, Zheng X, Kodama T, Ohashi Y. Marx line: fluorescein staining line on the inner lid as indicator of meibomian gland function. Am J Ophthalmol. (2006) 141:669-75. doi: 10.1016/j.ajo.2005.11.004

24. Rabensteiner DF, Aminfar H, Boldin I, Nitsche-Resch M, Berisha B, Schwantzer G, et al. Demodex mite infestation and its associations with tear film and ocular surface parameters in patients with ocular discomfort. Am J Ophthalmol. (2019) 204:7-12. doi: 10.1016/j.ajo.2019.03.007

25. Asbell PA, Stapleton FJ, Wickström K, Akpek EK, Aragona P, Dana R, et al. The international workshop on meibomian gland dysfunction: report of the clinical trials subcommittee. Invest Ophthalmol Vis Sci. (2011) 52:2065-85. doi: 10.1167/iovs.10-6997h

26. Cho BJ, Jee DH, Kim WJ, Shin MC, Kim EC, Kim MS, et al. Direct visualization of continuous meibum secretion from the orifices of meibomian glands to the tear film. Cornea. (2019) 38:1245-52. doi: 10.1097/ICO.0000000000002049

27. Utsunomiya T, Kawahara A, Hanada K, Yoshida A. Effects of diquafosol ophthalmic solution on quality of life in dry eye assessed using the dry eye-related quality-of-life score questionnaire: effectiveness in patients while reading and using visual display terminals. Cornea. (2017) 36:908-14. doi: 10.1097/ICO.0000000000001241

28. Guo L, Yang J, Mai J, Du X, Guo Y, Li P, et al. Prevalence and associated factors of myopia among primary and middle school-aged students: a school-based study in Guangzhou. Eye (Lond). (2016) 30:796-804. doi: 10.1038/eye.2016.39

29. Kim TH, Moon NJ. Clinical correlations of dry eye syndrome and allergic conjunctivitis in Korean children. J Pediatr Ophthalmol Strabismus. (2013) 50:124-7. doi: 10.3928/01913913-20130108-01

30. Coats DK, Paysse EA, Kim DS. Excessive blinking in childhood: a prospective evaluation of 99 children. Ophthalmology. (2001) 108:1556-61. doi: 10.1016/S0161-6420(01)00644-3

31. Argilés M, Cardona G, Pérez-Cabré E, Rodríguez M. Blink rate and incomplete blinks in six different controlled hard-copy and electronic reading conditions. Invest Ophthalmol Vis Sci. (2015) 56:6679-85. doi: 10.1167/iovs.15-16967

32. Park J, Baek S. Dry eye syndrome in thyroid eye disease patients: The role of increased incomplete blinking and Meibomian gland loss. Acta Ophthalmol. (2019) 97:e800-6. doi: 10.1111/aos.14000

33. Chou YB, Fan NW, Lin PY. Value of lipid layer thickness and blinking pattern in approaching patients with dry eye symptoms. Can J Ophthalmol. (2019) 54:735-40. doi: 10.1016/j.jcjo.2019.03.005

34. Ren Y, Chen J, Zheng Q, Chen W. Short-term effect of a developed warming moist chamber goggle for video display terminal-associated dry eye. BMC Ophthalmol. (2018) 18:33. doi: 10.1186/s12886-018-0700-y
35. Kawashima M, Tsubota K. Tear lipid layer deficiency associated with incomplete blinking: a case report. BMC Ophthalmol. (2013) 13:34. doi: 10.1186/1471-2415-13-34

36. Portello JK, Rosenfield M, Chu CA. Blink rate, incomplete blinks and computer vision syndrome. Optom Vis Sci. (2013) 90:482-7. doi: 10.1097/OPX.0b013e31828f09a7

37. Nakamura S, Kinoshita S, Yokoi N, Ogawa Y, Shibuya M, Nakashima $\mathrm{H}$, et al. Lacrimal hypofunction as a new mechanism of dry eye in visual display terminal users. PLoS ONE. (2010) 5:e11119. doi: 10.1371/journal.pone.0011119

38. Korb DR, Baron DF, Herman JP, Finnemore VM, Exford JM, Hermosa JL et al. Tear film lipid layer thickness as a function of blinking. Cornea. (1994) 13:354-9. doi: 10.1097/00003226-199407000-00012

39. Randolph SA. Computer vision syndrome. Workplace Health Saf. (2017) 65:328. doi: 10.1177/2165079917712727

40. Wang MTM, Tien L, Han A, Lee JM, Kim D, Markoulli M, et al. Impact of blinking on ocular surface and tear film parameters. Ocul Surf. (2018) 16:424-9. doi: 10.1016/j.jtos.2018.06.001

41. Georgiev GA, Eftimov P, Yokoi N. Structure-function relationship of tear film lipid layer: a contemporary perspective. Exp Eye Res. (2017) 163:17-28. doi: 10.1016/j.exer.2017.03.013

42. Jung SJ, Mehta JS, Tong L. Effects of environment pollution on the ocular surface. Ocul Surf. (2018) 16:198-205. doi: 10.1016/j.jtos.2018.03.001

Conflict of Interest: The authors declare that the research was conducted in the absence of any commercial or financial relationships that could be construed as a potential conflict of interest.

Publisher's Note: All claims expressed in this article are solely those of the authors and do not necessarily represent those of their affiliated organizations, or those of the publisher, the editors and the reviewers. Any product that may be evaluated in this article, or claim that may be made by its manufacturer, is not guaranteed or endorsed by the publisher.

Copyright (C) 2021 Zhao, Wu, Cheng, Xiao, Shu, Ge, Tian and Shao. This is an open-access article distributed under the terms of the Creative Commons Attribution License (CC BY). The use, distribution or reproduction in other forums is permitted, provided the original author(s) and the copyright owner(s) are credited and that the original publication in this journal is cited, in accordance with accepted academic practice. No use, distribution or reproduction is permitted which does not comply with these terms. 